\title{
Report (1966-1970) of the Subcommittee on Streptococci and Pneumococci to the International Committee on Nomenclature of Bacteria
}

14 August 1970

Centro Médico, Mexico City, Mexico

Since 1966, the Subcommittee on Streptococci and Pneumococci engaged in several studies proposed at the meetings in Moscow (Int. J. Syst. Bacteriol. 17:281-314). Progress on these studies is described below.

New provisional M-types of Streptococcus pyogenes Group A. Provisional M-types 52 through 61 were assigned to strains of Group A streptococci submitted for confirmation. Streptococcal reference centers in London, Atlanta, Prague, and New York City participated in laboratory studies and agreed that criteria for provisional designation of new M-types were fulfilled. Studies were extended to demonstrate the feasibility of producing specific absorbed $M$-antisera for each new type and to recognize their occurrence in different geographic areas and clinical situations.

Second International Streptococcal Type Distribution Survey. The Second International Streptococcal Type Distribution Survey was conducted under the direction of W. Köhler. Fifteen countries participated. Representative strains of Group A streptococci collected from cases of pharyngitis, scarlet fever, and other streptococcal illnesses in each country were serotyped by $\mathrm{T}$-agglutination and, if possible, M-precipitin tests. The distribution of types in relation to geographic areas and kind of illness was determined.
Cooperative study of national reference laboratories in Streptococcus typing. J. Rotta, Director of the World Health Organization's International Reference Centre for Streptococcus Typing, coordinated a study in which 15 reference laboratories typed and exchanged strains of Group A streptococci representing many serotypes. The purpose of the study was to compare the typing reactions obtained for identical strains in different laboratories.

Streptococcal strains for producing grouping antisera. Streptococcal strains used in various laboratories for producing antisera for groups $\mathrm{B}, \mathrm{C}, \mathrm{D}, \mathrm{G}$, and $\mathrm{N}$ were studied to determine which strains might be recommended for the routine production of antiserum to obtain uniformity of grouping reactions among streptococcus laboratories.

Classification of nonhemolytic streptococci. Several investigators studied strains of Streptococcus mutans and a variety of viridans and nonhemolytic streptococci to develop improved schema for identifying and classifying these organisms.

Pneumococcal types in Denmark. Information on pneumococcal types isolated from blood, spinal fluid, and pleural exudate during 1954-1969 in Denmark was compiled.

Max D. Moody, Secretary R. E. O. Williams, Chairman 